\title{
Transverse momentum distributions of charged particles in pp and nuclear collisions with ALICE at the LHC
}

\author{
Edgar Perez Lezama*t \\ GSI Helmholtzzentrum für Schwerionenforschung \\ Johann Wolfgang Goethe Universität \\ E-mail: eperezle@cern.ch
}

\begin{abstract}
The charged-particle transverse momentum spectrum in pp collisions is an important observable for testing perturbative QCD calculations and serves as a reference for heavy-ion collisions to study the properties of deconfined matter created in nucleus-nucleus (AA) collisions. The study of inclusive charged particle spectra gives information on parton energy loss in the medium created in AA collisions, leading to a suppression of hadron production at high transverse momentum $\left(p_{\mathrm{T}}\right)$. This effect can be investigated by calculating the nuclear modification factor, defined as the ratio between the $p_{\mathrm{T}}$ spectrum measured in nucleus-nucleus collisions and a reference spectrum in pp collisions scaled by the number of binary collisions. ALICE has measured pp collisions over a large energy range $\sqrt{\mathrm{s}}=13,7,5.02$ and $2.76 \mathrm{TeV}, \mathrm{p}-\mathrm{Pb}$ at $\sqrt{\mathrm{s}_{\mathrm{NN}}}=5.02 \mathrm{TeV}$, Xe-Xe at $\sqrt{\mathrm{S}_{\mathrm{NN}}}=5.44 \mathrm{TeV}$ and $\mathrm{Pb}-\mathrm{Pb} \sqrt{\mathrm{s}_{\mathrm{NN}}}=5.02 \mathrm{TeV}$ and $2.76 \mathrm{TeV}$. We show the $p_{\mathrm{T}}$ spectra in pp and nuclear collisions as well as the nuclear modification factors with an exceptional precision as compared to previous results.
\end{abstract}

The 39th International Conference on High Energy Physics (ICHEP2018)

4-11 July, 2018

Seoul, Korea

${ }^{*}$ Speaker.

${ }^{\dagger}$ for the ALICE Collaboration 


\section{Introduction}

The charged particle production has been measured in different collisions systems and in several experiments. The measurements of the transverse momentum spectra in pp and AuAu collisions at $\sqrt{s_{\mathrm{NN}}}=130 \mathrm{GeV}$, at RHIC experiments [1,2], have shown that the high- $p_{\mathrm{T}}$ particle production in heavy-ion collisions is suppressed compared to the production measured in pp. Bjorken proposed that suppression of jets or high- $p_{\mathrm{T}}$ particles is associated to parton energy loss in the hot and dense QCD medium created in the collision of heavy-ions [3], which leads to a modification of transverse momentum distributions of the particles in the final state. The ALICE experiment has observed a stronger suppression of hadron production at high- $p_{\mathrm{T}}$ in central $\mathrm{Pb}-\mathrm{Pb}$ collisions at $\sqrt{s_{\mathrm{NN}}}=2.76 \mathrm{TeV}$ [4] with respect to RICH at lower energies. This proves that a denser and hotter medium is created with respect to the medium created at RHIC energies. This observation is typically quantified in terms of the nuclear modification factor, defined as:

$$
R_{\mathrm{AA}}=\frac{1}{\left\langle T_{\mathrm{AA}}\right\rangle} \frac{\mathrm{d} N_{\mathrm{AA}} / \mathrm{d} p_{\mathrm{T}}}{\mathrm{d} \sigma_{p p} / \mathrm{d} p_{\mathrm{T}}}
$$

where $\mathrm{d} N_{\mathrm{AA}} / \mathrm{d} p_{T}$ and $\sigma_{p p}$ represent the yield in nucleus-nucleus collisions and the cross section in pp collisions, respectively. $\left\langle\mathrm{T}_{\mathrm{AA}}\right\rangle$ is the nuclear overlap function, expressed as the ratio of the number of binary nucleon-nucleon collisions $\left\langle\mathrm{N}_{\text {coll }}\right\rangle$ obtained from a Glauber model and the inelastic nucleon-nucleon cross section $\sigma_{\text {inel }}^{\mathrm{NN}}$. If the $\mathrm{Pb}-\mathrm{Pb}$ collisions are a simple superposition of elementary pp collisions the $R_{\mathrm{AA}}$ is expected to be equal to unity, while $R_{\mathrm{AA}}<1$ indicates a suppression of charged-particle production compared to binary-collision scaling.

\section{Analysis}

The results reported here are based on the analysis of $1.1 \times 10^{6} \mathrm{Xe}-\mathrm{Xe}$ collisions at $\sqrt{s_{\mathrm{NN}}}=$ $5.44 \mathrm{TeV}$ and $20 \times 10^{6} \mathrm{~Pb}-\mathrm{Pb}$ collisions at $\sqrt{s_{\mathrm{NN}}}=5.02 \mathrm{TeV}$ recorded with the ALICE detector during late 2017 and 2015, respectively. The primary charged particles are reconstructed using the ITS (Inner Tracking System) and the TPC (Time Projection Chamber). In Pb-Pb collisions the sum of amplitudes measured in V0A and V0C detectors is used to estimate the collision centrality [5]. Since ALICE did not record pp collisions at $\sqrt{s}=5.44 \mathrm{TeV}$, the pp reference was obtained using the power law interpolation method between the measurements at $\sqrt{s}=5.02 \mathrm{TeV}$ and $7 \mathrm{TeV}$. The scaling of the cross section at a fixed $p_{\mathrm{T}}$ is approximated to a power law in the collision energy, i.e. $\mathrm{d} \sigma / \mathrm{d} p_{\mathrm{T}}(\sqrt{s}) \propto \sqrt{s}^{n}$.

The corrections applied to the data are calculated using the combined information of MC simulations based on PYTHIA8 or HIJING generators, and data. To account for the differences in the particle composition between event generators and data, the charged-particle reconstruction efficiency $(\sim 70 \%)$ was calculated from the particle-dependent efficiencies weighted by the relative abundances of each particle measured in pp at $7 \mathrm{TeV}$ and $\mathrm{Pb}-\mathrm{Pb}$ collisions at $2.76 \mathrm{TeV}$. The correction for contamination by secondary particles, produced from weak decays or secondary interactions, is estimated using the transverse impact parameter distributions $\left(\mathrm{DCA}_{x y}\right)$ of particles in data and MC. This correction is important only at low $p_{\mathrm{T}}(\sim 8 \%)$, while the correction for $p_{\mathrm{T}}$-resolution is relevant only at high- $p_{\mathrm{T}}$. 


\section{Results}

The transverse momentum distribution for primary charged particles has been measured for $\mathrm{Pb}-\mathrm{Pb}$ and $\mathrm{Xe}-\mathrm{Xe}$ collisions at $\sqrt{s_{\mathrm{NN}}}=5.02 \mathrm{TeV}$ and $5.44 \mathrm{TeV}$, respectively. The differential cross section has been measured for INEL pp collision at $\sqrt{s}=5.02 \mathrm{TeV}$. The $R_{\mathrm{AA}}$ at central collisions 0-5\% has a minimum at $p_{\mathrm{T}}=6-7 \mathrm{GeV} / \mathrm{c}$ and an almost linear rise for larger momenta. Similar $p_{\mathrm{T}}$ dependence of $R_{\mathrm{AA}}$ is observed for $\mathrm{Pb}-\mathrm{Pb}$ and $\mathrm{Xe}-\mathrm{Xe}$ collisions, but the suppression of high-momentum particles is apparently stronger in $\mathrm{Pb}-\mathrm{Pb}$ for the same centrality class.

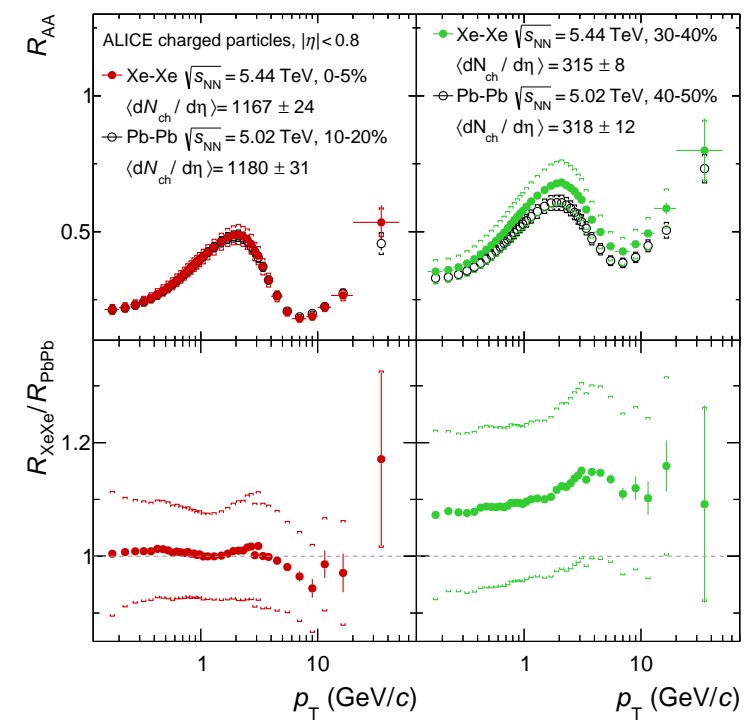

Figure 1: Comparison of nuclear modification factors in $\mathrm{Xe}-\mathrm{Xe}$ (full points) and $\mathrm{Pb}-\mathrm{Pb}$ (open points) collisions for similar ranges of mean multiplicity, 0-5\% (left) and 30-40\% (right) Xe-Xe centrality classes. The brackets represent the systematic plus normalization uncertainties and the vertical lines the statistical errors.

The nuclear modification factors from $\mathrm{Xe}-\mathrm{Xe}$ and $\mathrm{Pb}-\mathrm{Pb}$ collisions and their ratios at similar average multiplicity $\left\langle\mathrm{d} N_{\mathrm{ch}} / \mathrm{d} \eta\right\rangle$ are shown in Figure 1. In central Xe-Xe collisions $(0-5 \%)$, the average multiplicity is $\left\langle\mathrm{d} N_{\mathrm{ch}} / \mathrm{d} \eta\right\rangle=1167 \pm 24$, while for $\mathrm{Pb}-\mathrm{Pb}$ collisions in $10-20 \%$ centrality range is $\left\langle\mathrm{d} N_{\mathrm{ch}} / \mathrm{d} \eta\right\rangle=1180 \pm 31$. The ratio of the nuclear modification factor at similar average multiplicity (bottom panels of Fig.1) shows a remarkable agreement between both systems. In the comparison of $30-40 \% \mathrm{Xe}-\mathrm{Xe}$ and $40-50 \% \mathrm{~Pb}-\mathrm{Pb}$, the ratio is approximately flat and the systematic uncertainties cover the ratio at unity. The compatibility in the nuclear modification factors at similar values of average multiplicity is in agreement with results from the study of fractional momentum loss of high- $p_{\mathrm{T}}$ partons at RHIC and at the LHC. The ratios at similar multiplicities supports the idea that the $R_{\mathrm{AA}}$ ratios in the same centrality classes is not a meaningful comparison, since the average multiplicities or the medium sizes are not comparable between the two systems. A comparison of the $R_{\mathrm{AA}}$ as a function of $\left\langle\mathrm{d} N_{\mathrm{ch}} / \mathrm{d} \eta\right\rangle$ in $\mathrm{Xe}-\mathrm{Xe}$ and $\mathrm{Pb}-\mathrm{Pb}$ collisions for three different regions of $p_{\mathrm{T}}$ (low, medium, and high) is shown in Figure 2. A remarkable agreement in the $R_{\mathrm{AA}}$ is observed between $\mathrm{Xe}-\mathrm{Xe}$ and $\mathrm{Pb}-\mathrm{Pb}$ collisions when compared at similar multiplicity ranges, for $\left\langle\mathrm{d} N_{\mathrm{ch}} / \mathrm{d} \eta\right\rangle>400$. The observed similarity in the $R_{\mathrm{AA}}$ at high- $p_{\mathrm{T}}$ is consistent with the 


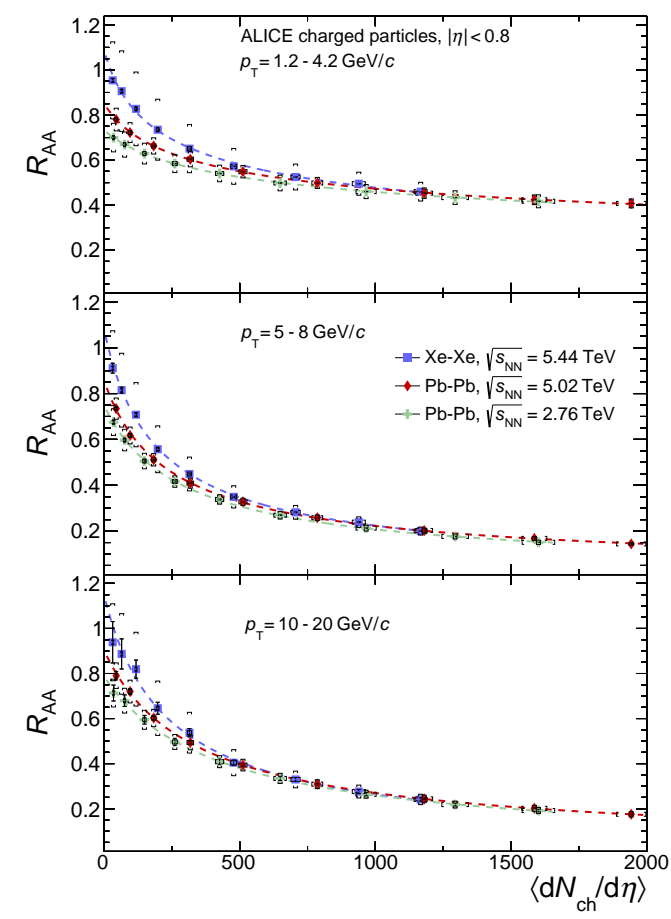

Figure 2: Comparison of the nuclear modification factor in $\mathrm{Xe}-\mathrm{Xe}$ and $\mathrm{Pb}-\mathrm{Pb}$ collisions integrated over three $p_{\mathrm{T}}$ regions as a function of $\left\langle\mathrm{d} N_{\mathrm{ch}} / \mathrm{d} \eta\right\rangle$. The horizontal bars correspond to the RMS of the distribution in each bin. The dashed line is a power law fit to the data only for guidance purposes.

quadratic path length dependence of medium induced radiative energy loss, assuming a simplified radiative energy loss scenario and when assuming identical medium thermalization times.

\section{Summary}

The charged particle transverse momentum distributions have been measured for $\mathrm{pp}$ and $\mathrm{Pb}-\mathrm{Pb}$ collisions at $\sqrt{s_{\mathrm{NN}}}=5.02 \mathrm{TeV}$ and Xe-Xe collisions at $\sqrt{s_{\mathrm{NN}}}=5.44 \mathrm{TeV}$. The ratio of $R_{\mathrm{AA}}$ between $\mathrm{Pb}-\mathrm{Pb}$ and $\mathrm{Xe}-\mathrm{Xe}$ shows a good agreement for multiplicities of $\left\langle\mathrm{d} N_{\mathrm{ch}} / \mathrm{d} \eta\right\rangle \approx 1170$. The compatible $R_{\mathrm{AA}}$ in both systems at high- $p_{\mathrm{T}}$ is consistent with a quadratic path length dependence of medium energy loss.

\section{References}

[1] PHENIX Collaboration 2002 Phys. Rev. Lett. 88022301

[2] STAR Collaboration 2002 Phys. Rev. Lett. 89022301

[3] Bjorken J D 1982 FERMILAB-PUB-82-059-THY

[4] ALICE Collaboration 2013 Phys. Lett. 88 52-62.

[5] ALICE Collaboration 2013 Phys. Rev. C 88044909. 